\title{
Analysis of Environmental Swipes Using Fluorescence Microscopy- Summary Report
}

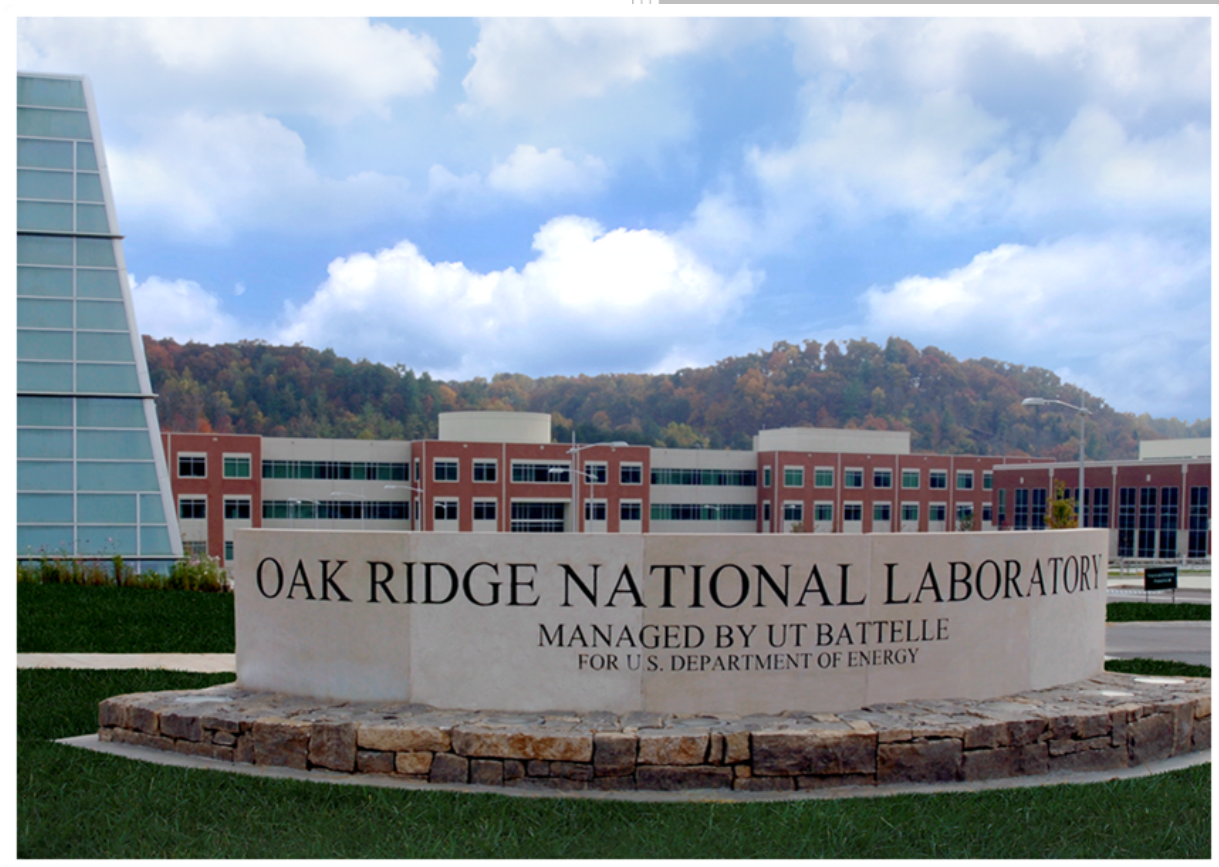

Approved for public release. Distribution is unlimited.

Robert C. Dunn Heath A. Huckabay Denise L. Lee Brian W. Ticknor

May 2017 


\title{
DOCUMENT AVAILABILITY
}

Reports produced after January 1, 1996, are generally available free via US Department of Energy (DOE) SciTech Connect.

Website http://www.osti.gov/scitech/

Reports produced before January 1, 1996, may be purchased by members of the public from the following source:

\author{
National Technical Information Service \\ 5285 Port Royal Road \\ Springfield, VA 22161 \\ Telephone 703-605-6000 (1-800-553-6847) \\ TDD 703-487-4639 \\ Fax 703-605-6900 \\ E-mail info@ntis.gov \\ Website http://classic.ntis.gov/
}

Reports are available to DOE employees, DOE contractors, Energy Technology Data Exchange representatives, and International Nuclear Information System representatives from the following source:

Office of Scientific and Technical Information

PO Box 62

Oak Ridge, TN 37831

Telephone 865-576-8401

Fax 865-576-5728

E-mail reports@osti.gov

Website http://www.osti.gov/contact.html

This report was prepared as an account of work sponsored by an agency of the United States Government. Neither the United States Government nor any agency thereof, nor any of their employees, makes any warranty, express or implied, or assumes any legal liability or responsibility for the accuracy, completeness, or usefulness of any information, apparatus, product, or process disclosed, or represents that its use would not infringe privately owned rights. Reference herein to any specific commercial product, process, or service by trade name, trademark, manufacturer, or otherwise, does not necessarily constitute or imply its endorsement, recommendation, or favoring by the United States Government or any agency thereof. The views and opinions of authors expressed herein do not necessarily state or reflect those of the United States Government or any agency thereof. 
Nuclear Security and Isotope Technology Division

\title{
ANALYSIS OF ENVIRONMENTAL SWIPE USING FLUORESCENCE MICROSCOPY- SUMMARY REPORT
}

\author{
Robert C. Dunn,* Heath A. Huckabay, Denise L. Lee, and Brian W. Ticknor
}

* The University of Kansas

Date Published: May 2017

Prepared by

OAK RIDGE NATIONAL LABORATORY

Oak Ridge, TN 37831-6283

managed by

UT-BATTELLE, LLC

for the

US DEPARTMENT OF ENERGY

under contract DE-AC05-00OR22725 



\begin{abstract}
This report summarizes efforts as part of a collaboration between Oak Ridge National Laboratory (ORNL) and the University of Kansas (KU) during fiscal year 2016 for the purpose of screening environmental swipes (ESs) using fluorescence microscopy. The overall goal of this work is to image actinide-bearing species by exploiting the fluorescence properties of the uranyl moiety. If successfully developed, this capability would permit automated screening of actinide species in environmental swipes to prioritize samples for destructive analysis. The evaluation of efforts to date has shown that uranyl fluoride can be imaged using fluorescence microscopy and the vibronic structure can be resolved; however, difficulties arise when attempting to image these materials on ESs because of spectral interference. Due to the fluorescence decay differences between cellulosic fibers and uranyl-containing materials, time-gating approaches were investigated to minimize this interference. This approach shows promise; however, the technique requires additional developmental work before it can be transitioned to a robust method for laboratory use.
\end{abstract}

\title{
1. MISSION RELEVANCE
}

Environmental sampling and sample analyses by the International Atomic Energy Agency (IAEA) Network of Analytical Laboratories (NWAL) comprise a critical technical tool used by the IAEA to detect facility misuse under a Comprehensive Safeguards Agreement and to detect clandestine facilities and activities under an Additional Protocol. However, the number of samples that must be analyzed often creates bottlenecks that delay analysis and results reporting. Current ES prioritization relies on simple (and imprecise) gamma counting and consideration of subjective factors. Investigating new methods, therefore, may assist in improving initial ES characterization so that prioritization and sample handling can be based more firmly on specific content information.

To objectively prioritize environmental sample analysis and accelerate the completion of priority samples, the presence of nuclear material must be confirmed, and its location on the ES must be isolated to efficiently excise only the portion of the swipe of interest, thereby reducing dilution of the signal or (for particles) focusing the analysis. Previous work found that a fluorescence microscope with readily interchangeable filter sets and enhanced light-gathering optical instruments could potentially be used to evaluate the feasibility of fluorescence microscopy as a method for prescreening ES swipes, which must be maintained in their clear, double-bagged evidentiary layers. ${ }^{1}$

\section{TECHNICAL DESCRIPTION}

Fluorescence spectroscopy is a method that probes the emissions of light from certain materials. Emissions occur as these materials undergo electronic transitions. To describe this approach, consider a simplified Jablonski diagram of the possible transition processes that can occur in a material (Figure 1). In this technique, a target material is illuminated with a wavelength of light matching an electronic absorption band within the substance. Upon the absorption of a photon, an electron in the material is elevated from the $S_{0}$ to the $S_{1}$ excited electronic state. At this point, the molecule may undergo a number of processes, but the relevant event is the emission of a photon as the electron transitions from the $S_{1}$ to the $\mathrm{S}_{0}$ state, known as fluorescence. This photon will be of a longer wavelength than the excitation source (red-shifted) and can be filtered and subsequently detected for quantitative or imaging purposes. The fluorescence spectra obtained can be used to identify and quantify chemical species of safeguards interest. 

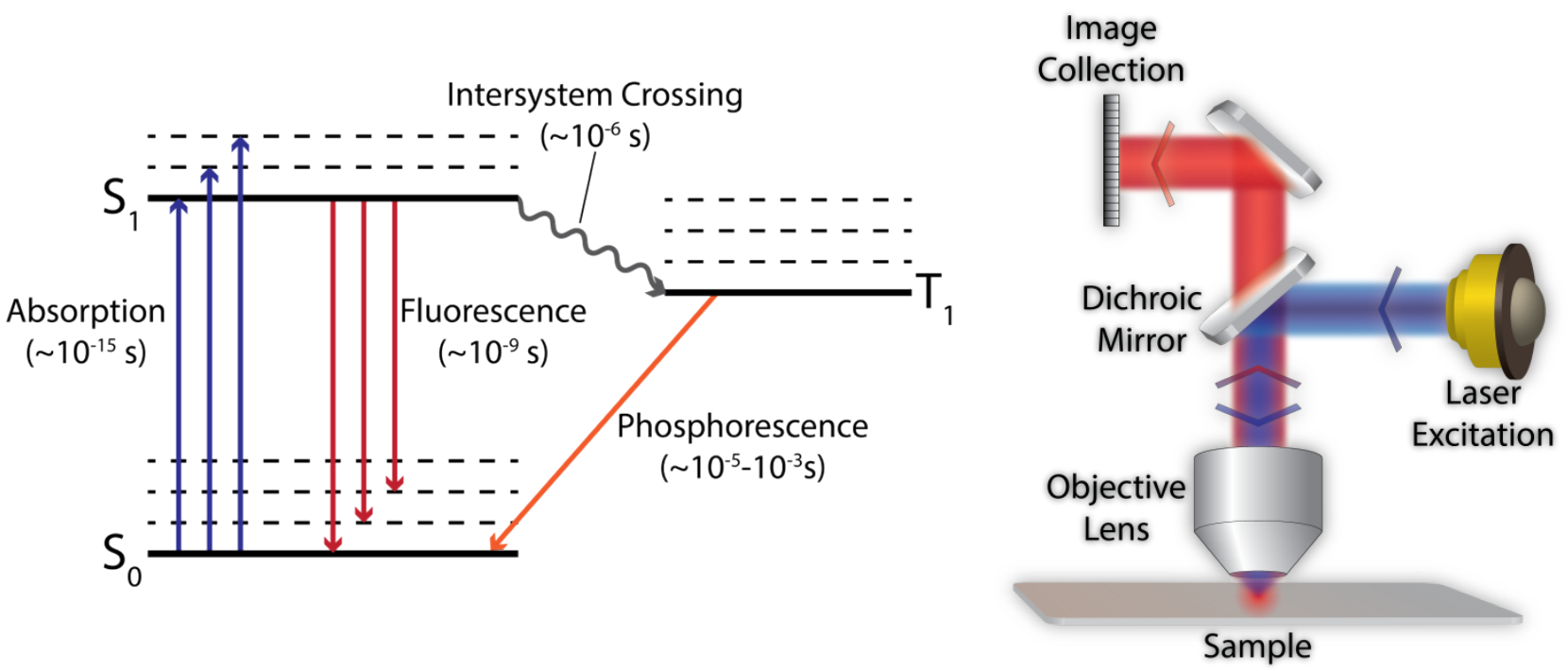

Figure 1. Shown at left is a Jablonski diagram illustrating the absorption and emission processes in a fluorescent or phosphorescent material. Shown at right is a fluorescence microscope which takes advantage of red-shifted emission inherent in fluorescent materials to examine them on a substrate.

Fluorescence microscopy integrates this technique into microscope optics, similar to confocal Raman spectroscopy. As in that case, this integration increases the laser fluence on the sample while reducing the possible background contribution. The configuration also shares the drawback that multiple samples will need to be collected to measure the entire surface of the swipe sample. Detection limits for fluorescence microscopy can reach the single-molecule level; however, known interferences will be present for swipe analysis. Both the bag material and the cotton swipe fluoresce, requiring filtering techniques to be applied.

The safeguards utility of this technique is to scan for, identify, and quantify fluorescent molecules that have safeguards significance. Like Raman spectroscopy, this technique relies on the target's chemical identity; notably, the uranyl ion is fluorescent and can be readily detected by fluorescence spectroscopy.

\section{EXPERIMENTAL}

Emission spectra and images were collected using the custom-built microscope shown in Figure. 2. The imaging platform is built on a Zeiss Axiovert 100TV inverted microscope equipped with three separate ports for emission, collection, and detection. Excitation light from a laser diode source $(405 \mathrm{~nm}$, $150 \mathrm{~mW}$ ) is provided from above the sample using a custom-designed optics train. The flexible design

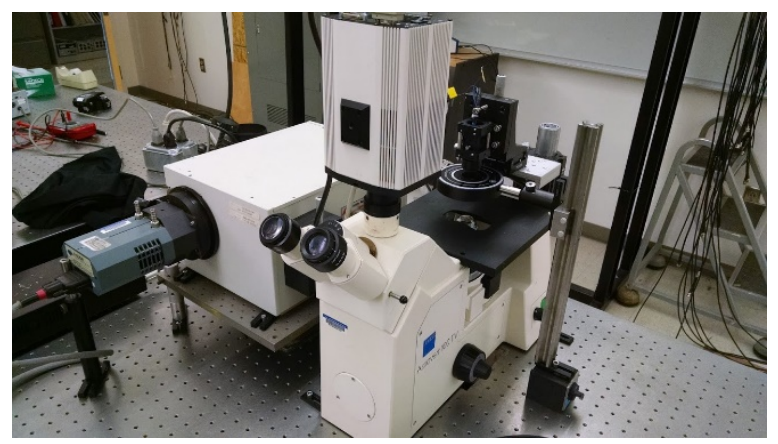

Figure 2. Inverted fluorescence microscope used to evaluate IAEA swipe emission screening.

Excitation light from a laser diode mounted above the sample stage focuses $405 \mathrm{~nm}$ light onto the sample. Sample emission is collected from below, filtered, and sent to one of two detection arms for emission imaging or spectral measurements. enables new excitation lines to be quickly evaluated. The excitation optics are mounted on a precision three-axis translation stage to center and focus the light 
in the field of view. For these experiments, a narrow bandwidth filter (Chroma HQ405/30) spectrally refined the excitation light and the imaging optics focused the excitation beam down to a diffractionlimited spot on the sample. The laser diode is powered using a Thor Labs LDC500 laser diode controller.

Emission from the sample is collected from below using a long working distance objective (Zeiss LD Achroplan 40×) and filtered to remove residual excitation light (Chroma HQ 490LP). For imaging experiments, sample emission is directed to a Photometrics CoolSnap K4 CCD camera (top port above eyepieces in Figure 2). Images are collected and analyzed using Micromanager and NIH Image software, respectively. For spectral measurements, sample emission is directed to the side port of the microscope, which is mated to an Acton Research SpectraPro 300i spectrograph equipped with an Andor DU420-BUcooled CCD camera (Figure 2). Spectra are collected and analyzed using MCD software from Andor Research Inc. All spectral measurements were calibrated using HeNe laser line sources at $543 \mathrm{~nm}$, $594 \mathrm{~nm}, 612 \mathrm{~nm}$, and $633 \mathrm{~nm}$ (Research Electro-Optics Inc.).

For time-gated experiments, a Uniblitz shutter (VS25S27M1R1) was inserted between the excitation source and sample as shown in Figure 2. Fast triggering of the Uniblitz shutter driver (VMM-DI) enabled $1 \mathrm{~ms}$ excitation pulses to be created. This approach was used to collect sample spectra at variable time delays following the excitation pulse. For these experiments, a four-channel pulse delay generator (Stanford Research, DG 535) was used to shape and control the timing between trigger pulses that initiated the shutter and those triggering the collection of spectra with the CCD camera. It should be noted that all components used in Figure 2 are commercially available and can be easily reconstructed in other labs, which was a design parameter for the project.

\section{RESULTS}

All swipes analyzed at KU were surrogate samples prepared at ORNL and double-bagged within two plastic bags (Ziploc type). Control experiments on empty plastic bags showed that they had good transparency at the excitation wavelength $(405 \mathrm{~nm})$ and negligible background emission (data not shown). This suggests that the plastic bag containment of samples produces minimal interference for emission measurements. The thickness of the bag (and sample swipes), however, does limit the numerical aperture (NA) of the microscope objective that can be used. This is because the NA of the system is related to the acceptance angle of light collected from the target specimen. Increasing this attribute improves resolution; however, this is generally achieved by decreasing the distance of the lens from the substrate. In order to acquire fluorescence measurements through the containment bags of the swipes, therefore, all experiments reported here were conducted with a long working distance microscope objective with modest numerical aperture $(40 \times, \mathrm{NA}=0.6)$.

\section{Emission Imaging of $\mathrm{UO}_{2} \mathrm{~F}_{2}$ Powder on Glass:}

To validate the platform capabilities for uranyl compound emission detection and spectral characterization, initial studies focused on $\mathrm{UO}_{2} \mathrm{~F}_{2}$ powder in the absence of the cotton swipe. The powder was dispersed on a glass slide and double-bagged in plastic bags. Figure 3 shows representative emission images of $\mathrm{UO}_{2} \mathrm{~F}_{2}$ particulates on the glass slide taken through the two plastic bags. Strong emission is observed from the particles following $405 \mathrm{~nm}$ excitation. The images in Figure 3, for example, were collected with $50 \mathrm{~ms}$ integration times. The $\mathrm{UO}_{2} \mathrm{~F}_{2}$ particles are photostable with minimal

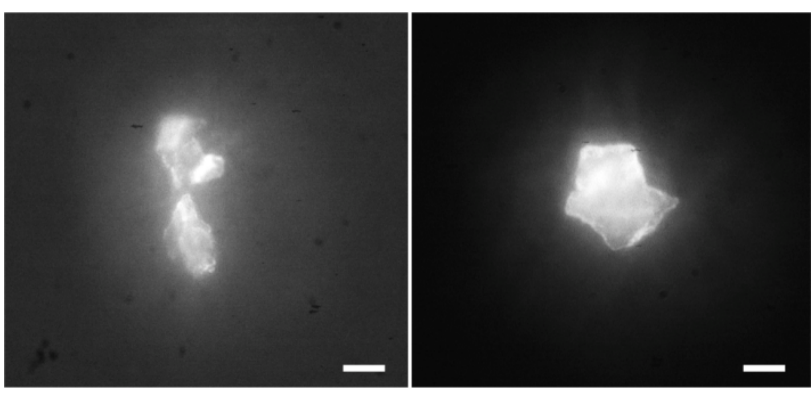

Figure 3. Representative emission images of $\mathrm{UO}_{2} \mathrm{~F}_{2}$ solids on glass, sealed within two plastic bags. Scale bars in each image represent $\mathbf{1 0}$ microns. 
photobleaching occurring during the timescale of the experiment. Strong emission and weak photobleaching improve the chances of developing a viable method for screening IAEA swipes.

Spectral Measurements of $\mathrm{UO}_{2} \mathrm{~F}_{2}$ Powder on Glass:

To confirm the emission source, spectral measurements were taken on the powdered samples. $\mathrm{UO}_{2} \mathrm{~F}_{2}$ emission exhibits a distinctive vibronic structure that may be helpful in fingerprinting samples. Figure 4 shows a typical spectrum collected on the powdered $\mathrm{UO}_{2} \mathrm{~F}_{2}$ sample. A distinctive vibronic structure is observed with six peaks resolved in the spectrum from $495 \mathrm{~nm}$ to $700 \mathrm{~nm}$. The high-energy side of the spectrum is near the emission filter cut-off (490 nm long pass), which distorts the bandshape somewhat. The location and relative intensities of the peaks in Figure 4 closely agree with previously reported spectra, thus confirming $\mathrm{UO}_{2} \mathrm{~F}_{2}$ as the origin of the emission. ${ }^{2-4}$

Spectral Measurements of Surrogate IAEA Swipes: After validating the ability to detect and spectrally characterize $\mathrm{UO}_{2} \mathrm{~F}_{2}$, experiments on the surrogate IAEA swipes were initiated. The samples consist of material transferred onto cotton swipes that are sealed within the double plastic bags. Background emissions from the cotton swipes are the main obstacle to developing a viable screening protocol based on sample emission. Figure 5, for example, shows the background spectra of a blank cotton swipe sealed within two plastic bags following $405 \mathrm{~nm}$ excitation. The abrupt rise in signal near $490 \mathrm{~nm}$ is due to the $490 \mathrm{~nm}$ long pass filter placed in the emission path to remove as much of this background as possible, without interfering with the spectral profile of $\mathrm{UO}_{2} \mathrm{~F}_{2}$ (Figure 4). The background emission in Figure 5 is artificially peaked near $500 \mathrm{~nm}$ due to the emission filter and slowly decays to the red, extending past $700 \mathrm{~nm}$. This large signal represents the tail of the main spectral feature that is peaked to the blue and, unfortunately, covers the entire spectral region of interest.

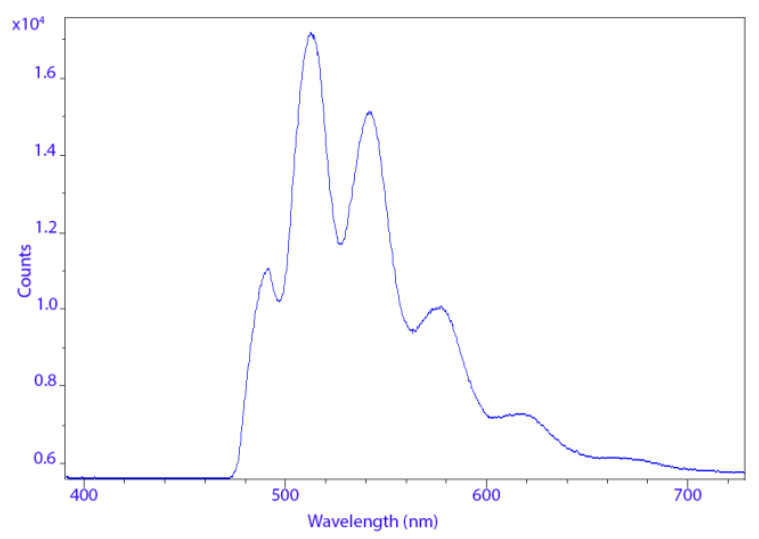

Figure 4. Representative emission spectrum of $\mathrm{UO}_{2} \mathrm{~F}_{2}$ following $405 \mathrm{~nm}$ excitation, collected with the microscope shown in Figure 1. A distinct vibronic structure is observed which agrees with previously reported spectra.

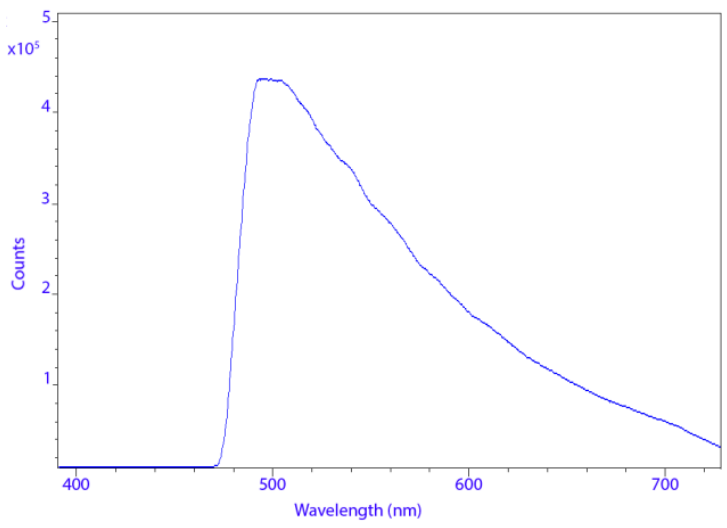

Figure 5. Emission spectrum of a blank cotton swipe sealed within two plastic bags following $405 \mathrm{~nm}$ excitation. Significant background emission is observed throughout the visible region, overlapping the spectrum of $\mathrm{UO}_{2} \mathrm{~F}_{2}$ as shown in Figure 4. The sharp rise in emission near $490 \mathrm{~nm}$ arises from long pass filters inserted in the optics train to reduce background.

Figure 6 shows a similar spectrum collected on a

double-bagged swipe dosed with $1.96 \mathrm{~g}$ of $\mathrm{UO}_{2} \mathrm{~F}_{2}$. The spectral profile is identical to the background emission measured in Figure 5. These and similar experiments performed on the other dosed swipes $\left(\mathrm{UO}_{2} \mathrm{~F}_{2}\right.$ in water, uranyl nitrate, etc.) exhibited similar profiles, illustrating the dominance of the background emission from the cotton swipe. Close inspection of the emission signals measured in Figures 5 and 6 reveals small, but repeatable, structure in the emission signals. While this band structure resembles the vibronic structure of $\mathrm{UO}_{2} \mathrm{~F}_{2}$, it arises from the spectral profile of the interference filter used to spectrally refine the emission. 


\section{Background Reduction Using Long Wavelength} Excitation: Since cotton absorbs in the ultraviolet, it is expected that background emission from ESs can be reduced by tuning the excitation light to longer wavelengths. Experiments similar to those shown in Figures 5 and 6 were therefore conducted using a $450 \mathrm{~nm}(1.6 \mathrm{~W})$ laser diode excitation source.

However, no significant improvements were observed. Further tuning of the excitation light to the red to reduce background is not feasible given the spectral characteristics of the uranyl compounds of interest. These experiments therefore suggest that it will be very difficult to separate out the emission from uranyl compounds against the large background of the cotton swipe using steady-state spectral measurements alone.

\section{Background Reduction Using Time-Gating: Time-} gating emission signals have proven to be an effective approach for minimizing unwanted background

interferences. ${ }^{5}$ This approach is optimal when the species of interest and the background have significantly different lifetimes, which is the case for uranyl compounds and cotton swipes. These differences are due to the separate mechanisms in which cotton and the uranyl species undergo emission: Cellulose fibers generally undergo direct radiative relaxation between two electronic states, whereas actinide species are currently thought to emit via a ligand-to-metal charge transfer transition. ${ }^{6}$ Selection rules between these two processes differ, which results in prompt emission from cotton swipes, lasting nanoseconds, and long lifetimes for uranyl compounds, lasting microseconds to milliseconds. ${ }^{7} \mathrm{By}$ delaying the collection of spectra following pulsed excitation, it should be possible to enhance the contribution from the uranyl species relative to the background from the cotton. To evaluate the effectiveness of time-gating the emission, the excitation source was pulsed and the spectrum collected with variable time delays by gating the CCD camera.

Initial experiments used excitation pulses generated by electronically modulating the laser diode drive current. After exploring a number of conditions, however, this method proved unsatisfactory. There was a small but significant amount of tailing in the excitation pulse, which complicated the time-gated collection of emission. To create a clean excitation pulse, a fast shutter was inserted in the excitation optics path, as shown in Figure 2. While this approach creates square wave excitation pulses, the time resolution is limited by the shutter speed ( $1 \mathrm{~ms}$ pulse width). It should be noted that these experiments were done with equipment on hand, which was not necessarily designed for time-resolved measurements. As such, while the results here are valid as a guide for future work, the details are not considered quantitative. Future work using pulsed laser sources combined with an intensified CCD camera that is made for time-resolved collection would provide a more complete exploration of important experimental conditions. ${ }^{5}$

Initial experiments validated the time-gating approach using the $\mathrm{UO}_{2} \mathrm{~F}_{2}$ powder samples on glass. Figure 7 compares the spectrum of $\mathrm{UO}_{2} \mathrm{~F}_{2}$ on glass at time delays of $16 \mathrm{~ms}$ and $17 \mathrm{~ms}$ following a $1 \mathrm{~ms}$ excitation at $405 \mathrm{~nm}$ using the mechanical shutter approach. These spectra can be compared with the steady-state spectrum shown in Figure 4. Clearly, measurable spectral features persist at significant delays following pulsed excitation of the $\mathrm{UO}_{2} \mathrm{~F}_{2}$ sample. The large spike seen in both spectra near $550 \mathrm{~nm}$ is an artifact due to a hot pixel in the camera. Other single-pixel spikes in the spectra represent cosmic events that can easily be removed using post-processing with the camera software. All spectra presented here, however, represent the raw, unprocessed data for complete evaluation of performance metrics. 

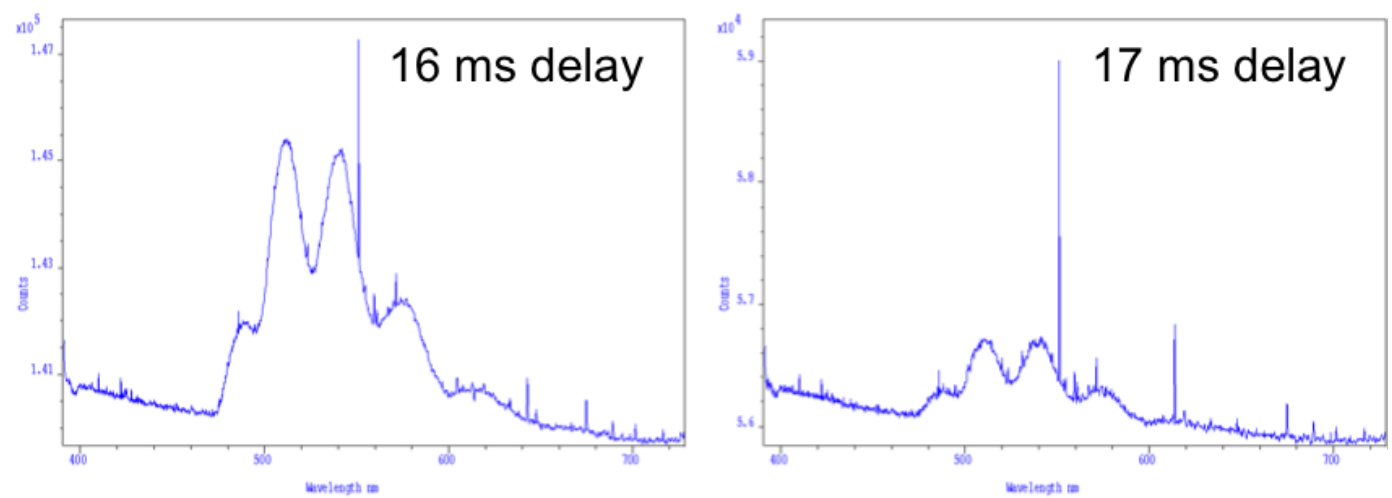

Figure 7. Spectra of $\mathrm{UO}_{2} \mathrm{~F}_{2}$ solids on glass at the indicated delays following pulsed excitation (1 ms) with $405 \mathrm{~nm}$ light.

Figure 8 compares spectra collected on a blank swipe to a swipe dosed with $\mathrm{UO}_{2} \mathrm{~F}_{2}(1.962 \mathrm{~g}) 17 \mathrm{~ms}$ following excitation. The $17 \mathrm{~ms}$ delay was selected as a balance between the intensity of uranyl fluorescence and the cotton background interference. At this time delay, the background emission from the blank is reduced but still present. The signal from the dosed swipe is slightly elevated compared with the blank and has slightly more structure; however, a clear differentiation cannot yet be made. Experiments continue to determine if significant differences exist between the blank and dosed samples at various time delays following excitation. Signal strength is a challenge in these experiments, and a highpowered diode laser has been ordered to increase excitation fluence in the $1 \mathrm{~ms}$ window provided by the mechanical shutter. With improved signal-to-noise, the origin of any spectral differences will be evaluated.
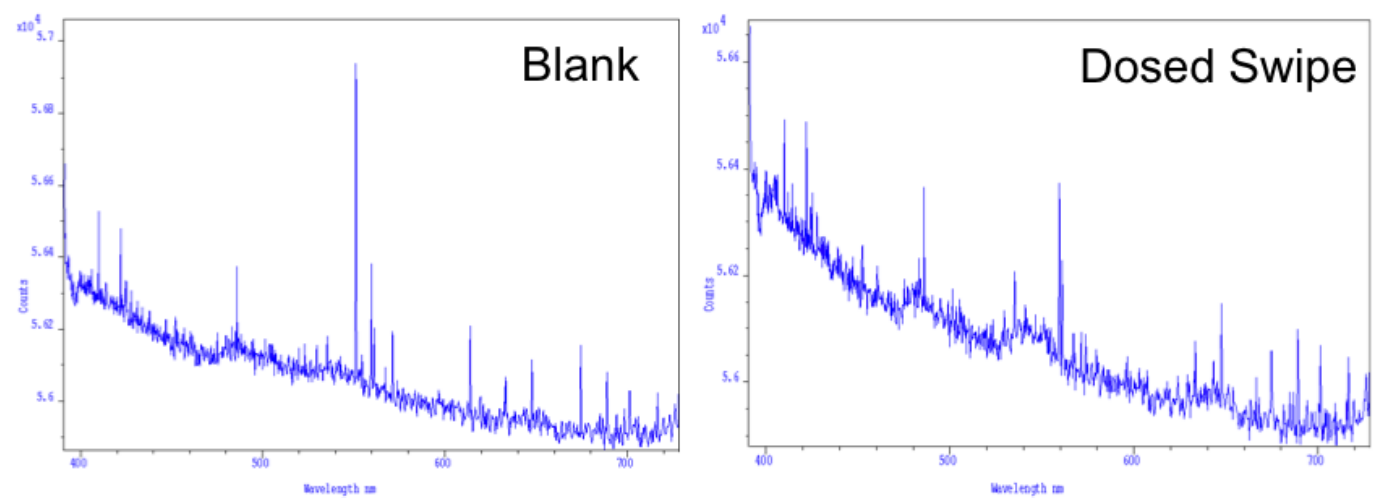

Figure 8. Spectra of a double-bagged blank swipe and one dosed with $\mathrm{UO}_{2} \mathrm{~F}_{2}$, collected at a delay of $17 \mathrm{~ms}$ following $1 \mathrm{~ms}$ excitation at $405 \mathrm{~nm}$.

\section{CONCLUSIONS AND FUTURE DIRECTIONS}

Time-gating the emission signal offers a promising approach for reducing background so that uranyl compound emissions from swipes can be detected and quantified. Experiments will continue once the high-powered laser arrives to explore improvements in signal-to-noise and the promise of time-gating the emission. 
In the future, a true pulsed excitation source coupled with an intensified CCD camera for time-resolved measurements would enable a full evaluation of IAEA swipe screening. A pulsed laser source will decrease the excitation pulse width while increasing peak power. Both are desirable for time-gating sample emissions. Moreover, an intensified CCD camera would optimize the collection of gated emission signals, enabling a full exploration of screening protocols. While current results suggest a potential screen for uranyl-containing species on collected ES samples, additional development would be required to move toward a robust analysis method for actinides. These studies may be performed pending interest in future development.

\section{REFERENCES}

1. Steeb, J. L.; Smith, N. A.; Lee, D. L.; Huckabay, H. A.; Ticknor, B. W. Technology Recommendations for Pre-screening of IAEA Swipe Samples, Progress Report; Argonne National Laboratory: Argonne, Illinois, 2015.

2. $\quad$ Gupta, S. K.; Dhobale, A. R.; Natarajan, V.; Godbole, S. V., Spectrochimica Acta Part A: Molecular and Biomolecular Spectroscopy 2014, 117, 204-209.

3. Beitz, J. V.; Williams, C. W., Journal of Alloys and Compounds 1997, 250 (1-2), 375-379.

4. Moulin, C.; Decambox, P.; Mauchien, P.; Pouyat, D.; Couston, L., Anal Chem 1996, 68 (18), 3204-3209.

5. Lakowicz, J. R., Principles of fluorescence spectroscopy. 3rd ed.; Springer: New York, 2006; p xxvi, $954 \mathrm{p}$.

6. Natrajan, L. S., Coordination Chemistry Reviews 2012, 256 (15-16), 1583-1603.

7. Bonhoure, I.; Meca, S.; Marti, V.; Pablo Joan, d.; Cortina, J.-L., Radiochimica Acta 2007, 95 (3), 165. 